\title{
Experimental observation of nonlinear pulse compression in nonuniform Bragg gratings
}

\author{
N. G. R. Broderick, D. Taverner, D. J. Richardson, M. Ibsen, and R. I. Laming \\ Optoelectronics Research Centre. Eniversity of Southampton. Southampton SO171BJ.L'K
}

\begin{abstract}
We demonstrate a scheme for optical pulse compression by cross-phase modulation that utilizes a nonuniform Bragg grating to work in reflection. Our scheme is similar to the conventional optical pushbroom, which works in transmission. This reflection geometry has the advantage of allowing the compressed signal to be observed easily, as it is spatially separate from the pump: This is to our knowledge the first nonlinear effect to be observed that requires a nonuniform grating.
\end{abstract}

The optical pushbroom ${ }^{1,2}$ is a scheme for pulse compression that combines the cross-phase modulation (XPM) of a strong pump pulse with the large group-velocity dispersion of a Bragg grating. In the original scheme the compressed probe is spatially located on the leading edge of the pump, and the compression effect is viewed in transmission, which makes experimental observation difficult, especially if the wavelength separation between pump and probe is small. For example, in the first demonstration of the optical pushbroom more than $80 \mathrm{~dB}$ of differential filtering between the pump and probe was required. ${ }^{3}$ Perhaps the most obvious way to overcome this problem is to separate the pump and the probe spatially. As optical fiber Bragg gratings are one dimensional, the only way that this separation can occur is if the probe is reflected while the pump is transmitted. Such a scheme is demonstrated here for what is believed to be the first time.

The essential difference between our geometry and de Sterke's ${ }^{1}$ is that our experiments are performed in a nonuniform Bragg grating (NBG) rather than in a uniform grating. Two properties of a $\mathrm{NBG}^{4}$ are crucial. First, the width of the photonic bandgap is a function of position. Second, light can penetrate significantly farther into a NBG than into a uniform grating before being reflected. These properties can be seen in Fig. 1, which shows the band diagram ${ }^{5}$ for the NBG used in our experiment. The hatched region shows the photonic bandgap as a function of position. Light outside this hatched region can propagate freely and is reflected at the interface. As in a uniform grating the group velocity is a strong function of the vertical distance from the band edge.

The two labeled regions in Fig. 1 indicate where the original and the new versions of the optical pushbroom operate. Region 1, below the bandgap, is where the original optical pushbroom works. Here the grating is totally transmissive and highly dispersive. Our experiments operate in region 2 , above the local bandgap, where the grating is highly reflective. although light can propagate through nearly $50 \%$ of the length of the grating before being reflected. In describing our scheme it is best to recall first the basic principles of the optical pushbroom.

Below the bandgap (region 1 in Fig. 1), although the grating is transmissive, light propagates with a significantly reduced group velocity. A weak cw probe situated in this region has a resonant field structure within the grating, allowing energy to be stored. When the strong pump enters the NBG it first encounters the back of the probe. Through XPM the probe's frequency is lowered, causing its speed to increase. The increased velocity allows the back of the probe to remain on the pump's leading edge, where it experiences still further XPM. As the pump continues to propagate it sweeps up more and more of the probe's energy until it leaves the grating. At the output one sees a narrow compressed pulse sitting on the leading edge of the pump.

Leaving the conventional pushbroom, we consider now the effects of XPM on light in region 2 of Fig. 1. Light at these frequencies can be thought of as being in a resonant cavity with a high-reflectivity mirror at one end. This setup allows significant energy to be stored in the grating. As before, the effect of the pump is to lower the frequency of the probe. The magnitude of this frequency shift is determined by the interaction time between any particular part of the probe and the leading edge of the pump. This time is determined by the distance between where part of the probe first saw the pump and where the probe is reflected. Clearly this time is greater for the back of the probe than for the front, and thus there

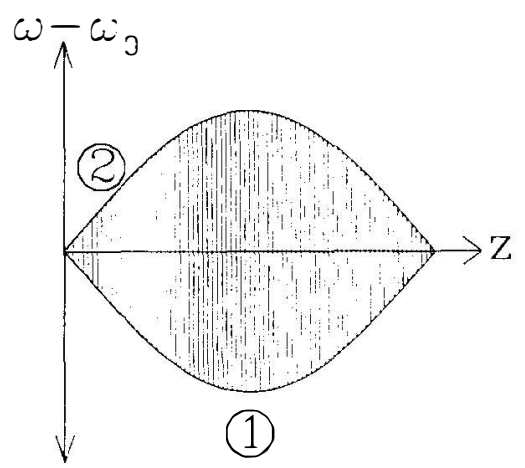

Fig. 1. Band diagram for the nonuniform grating used in the experiments. The hatched region indicates those frequencies (as a function of position) that are reflected by the grating. The conventional optical pushbroom works below the bandgap in region 1. Our alternative works above the bandgap in region 2 . 


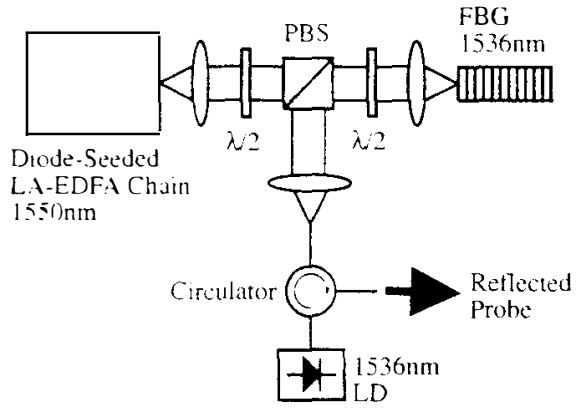

Fig. 2. Schematic of the experimental setup: PBS, polarization beam splitter: LA-EDFA. large-mode-area erbiumdoped fiber amplifier chain; FBG. fiber Bragg grating; LD, laser diode.

is a frequency chirp across the probe, where the front of the probe has a higher frequency than the back. Importantly, in the NBG under consideration higher frequencies are reflected later than lower frequencies. On reflection the chirp on the probe and the grating-induced chirp cancel. producing a compressed pulse containing much of the probe's energy. In the reflection ones sees a narrow spike followed by a long dip, which is the characteristic feature of the optical pushbroom. ${ }^{3}$ It should be noted that, unlike in the earlier XPM switching experiments in gratings by LaRochelle et al., which utilized a cw pump, in our experiments a pump pulse is necessary. This is due to the fact that we are altering the actual frequency of the pulse, which requires an intensity gradient across the pump.

Our experimental setup is shown in Fig. 2; 10-ns square pulses from a directly modulated distributedfeedback laser with a repetition rate of $4 \mathrm{kHz}$ and a wavelength of $1550 \mathrm{~nm}$ are amplified to high powers $(>10 \mathrm{~kW})$ by an erbium fiber amplifier chain.' Because of gain saturation the final pump shape is approximately triangular, with a 30-ps rise time and a 3-ns half-width see the inset in Fig. 4, below). The $\mathrm{cw}$ probe $(\approx 1 \mathrm{~mW})$ had a wavelength of $\approx 1536 \mathrm{~nm}$ and was coupled into the system through port 1 of the fiberized circulator (allowing easy detection of the reflected signal through port 3). Both the probe and the pump could be continuously temperature tuned over a 1-nm range, which allowed the probe to scan across the bandgap of the grating while, as expected, our results were insensitive to the precise pump wavelength. The spectral widths of the pump and the probe were measured to be $\approx 1.2 \mathrm{GHz}$ and $<100 \mathrm{M} \mathrm{Hz}$, respectively.

The reflection spectrum of the NBG used in our experiments is shown in Fig. 3. The 8-cm-long NBG had a $\sin x$ profile (see Fig. 1), with a width of $3.8 \mathrm{GHz}$ and a peak reflectivity of $98 \%$. The grating was holographically written into a germanosilicate fiber with a mode area of $30 \mu \mathrm{m}^{2}$. The dashed curve in Fig. 3 shows the theoretical reflection profile for a grating with the same parameters. Apart from the slight asymmetry on the short-wavelength side (the cause of which is unknown) the overall agreement is excellent. The grating was mounted in a section of capillary tube and angle polished at both ends so that reflections from the grating end faces were eliminated.
The pump and the probe were coupled into the NBG via a polarization beam splitter and were thus orthogonally polarized. The $\lambda / 2$ wave plate before the beam splitter allowed control of the pump power. The second $\lambda / 2$ wave plate, before the $\mathrm{NBG}$, ensured that the light's polarization axes matched the birefringence axes of the NBG.

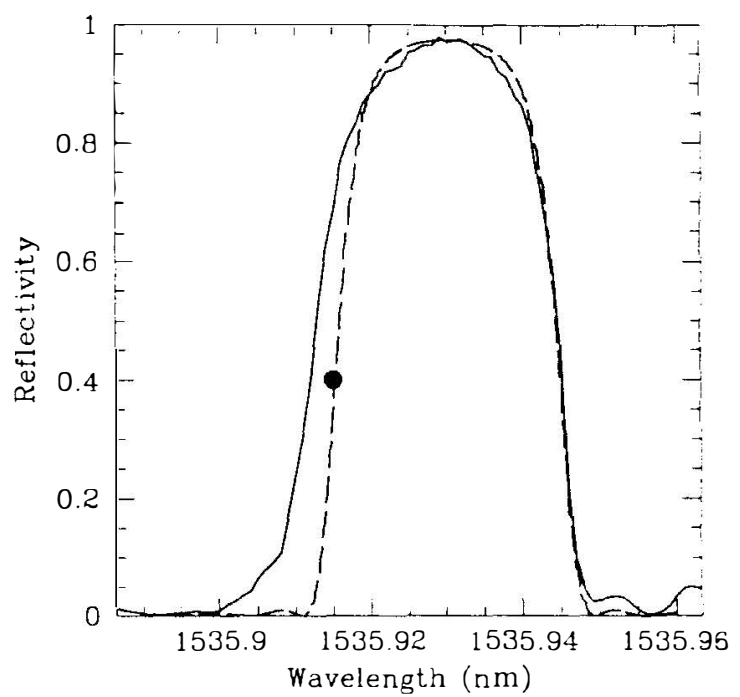

Fig. 3. Reflection spectra of the NBG used in the experiments (solid curve) and in the simulations (dashed curve). The wavelength resolution is $0.001 \mathrm{~nm}$. Note the very slight asymmetry in the actual spectrum and the almost complete absence of sidelobes. The grating bandwidth is $\approx 3.8 \mathrm{GHz}$. The filled circle indicates the frequency used in our numerical simulations.

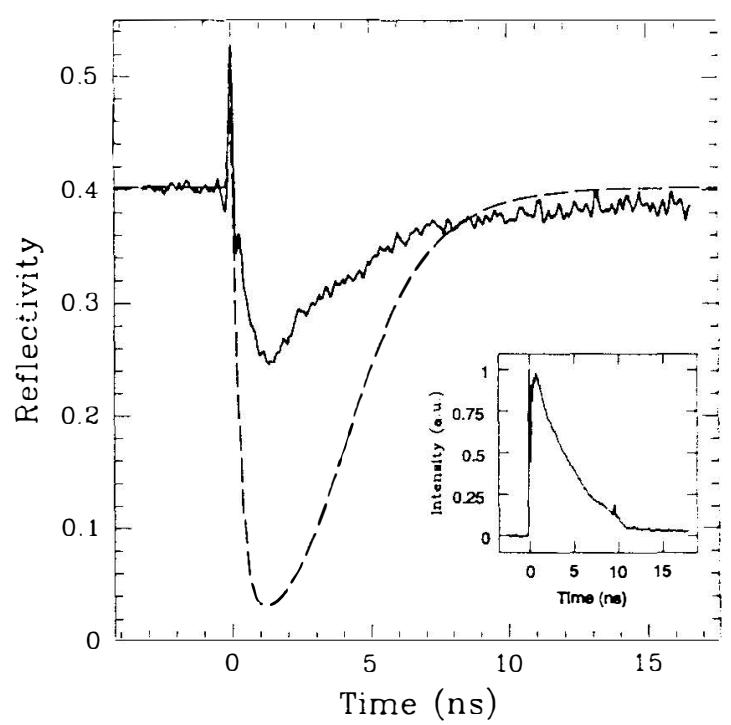

Fig. 4. Experimental trace of the reflected pushbroom. The solid curve shows the reflected fraction of the probe beam. The dashed curve shows the results of our numerical simulations of the system with the same parameters. Note the excellent agreement in the shape of the dip and the width of the front feature. The inset shows the intensity profile of the pump pulse before the grating. The time origins on the graphs are unrelated. The peak pump intensity is $\approx 10 \mathrm{GW} / \mathrm{cm}^{2}$. 
Tuning to the short-wavelength side of the grating, where the reflectivity was $40 \%$, we observed the reflected signal with a fiberized $\mathrm{p}-\mathrm{i}-\mathrm{n}$ photodiode with a rise time of $<50$ ps. A typical trace is shown in Fig. 4 (solid curve), in which the behavior described above can be clearly seen. Note that the reflectivity increases quickly to $55 \%$ and then drops to $25 \%$ before slowly rising again to the linear level. The inset shows the intensity profile of the pump pulse measured before the grating; the time origins on the two plots are unrelated. The dashed curve in Fig. 4 shows the results from numerical modeling of the experiment. Although our model does not predict the correct heights it does give the correct widths, and the overall agreement is very good, given that we used an idealized grating profile and approximated the pump as half a sech pulse. A full set of measurements of the reflected optical pushbroom when both the probe wavelength and the pump power were varied was performed; however, because of space considerations it is not presented here.

A comparison between our results and those for the conventional pushbroom ${ }^{1,3}$ reveals striking similarities in the temporal evolution of the signal. In both cases a sharp spike that contains much of the energy in the grating is followed by long dip caused by a combination of XPM-induced switching of the bandgap ${ }^{6}$ and the linear response time of the grating. Furthermore. in both cases the compression is caused by the interaction between the dispersion of the grating and the pumpinduced chirp of the probe. These similarities are sufficient, we think, to justify referring to our work as an optical pushbroom despite the differences in geometry between our work and that of de Sterke.

A drawback to our geometry is that the contrast between the maximum and minimum signals is lower in our case (contrast ratio. 2) than that seen in transmission $^{3}$ (where the ratio can easily be as high as 20). The smaller ratio is due to two factors: The interaction time between the pump and probe is shorter in our geometry, and less energy is stored. This drawback is offset by the comparative ease of detecting the reflected pushbroom, as we do not need to filter out the pump.

In conclusion, we have proposed and demonstrated an alternative geometry for pulse compression in Bragg gratings. This geometry requires a nonuniform grating and works in reflection. The main advantage of our geometry is that the signal is spatially separated from the pump, allowing easy observations of the pushbroom. In our experimental demonstration we were able to achieve a contrast ratio of greater than 2 between the maximum and minimum reflected signals.
The results of numerical simulations of this experiment closely match the observed trace. The reflected pushbroom is not restricted to the grating profile used here, and in numerical simulations the pushbroom can be seen in a wide variety of NBG's. It is expected that future theoretical research will allow us to optimize the grating design for this effect.

We note that, although previous experiments were performed in nonuniform gratings, ${ }^{89}$ the reflected pushbroom is believed to be the first example of a nonlinear effect in Bragg gratings that requires a nonuniform Bragg grating. The research of Eggleton et al.' was done in a superstructure grating, which can be regarded as a uniform grating in many instances, ${ }^{10}$ and the effects that they observed can all be seen in a uniform grating. Both our experiments and those of Eggleton et al. ${ }^{9}$ highlight the advantages of using nonuniform grating $\equiv$ as the medium for nonlinear experiments. Nonuniform Bragg gratings provide large rapidly varying dispersion of short length scale, making them ideal for experimental purposes.

This work is funded in part by the Engineering and Physical Sciences Research Council Realising Our Potential Award. D. J. Richardson and R. I. Laming acknowledge the support of the Royal Society through the university research fellowship scheme.

\section{References}

1. C. M. de Sterke. ('pt. Lett. 17, 914 ; 1992).

2. M. J. Steel and C M. de Sterke. Phrs. Rev. A 49, 5048 (1994).

3. N. G. R. Broderick. D. Taverner. D. J. Richardson. M. Isben, and P. I. Laming. in Bragg Gratings, Photosensivity, cnd Poling in Glass Fibers and Waveguides: Asjlications and Fundamentals. OSA Technical Diges: Series (Optical Society of America, Washington, D.C. to be published .

4. N. Broderick and C. M. de Sterke. Phys. Rer. E 52, 4458 (1995).

5. J. E. Sipe, L. Po:adian, and C. M. de Sterke, J. Opt. Soc. Am. A 11, 130־ 1994).

6. S. LaRochelle. Y. Hibino, V. Mizrahi, and G. I. Stegeman, Electron. Lett. 26, 1459 (1990).

7. D. Taverner, D. J. Richardson. L. Dong, J. E. Caplen, K. Williams, anci R. V. Penty. Opt. Lett. 22, 378 (1997).

8. U. Mohideen. R E. Slusher. V. Mizrahi, T. Erdogan, J. E. Sipe, M. Gonokami. P. J. Lemaire, C. M. de Sterke, and N. G. R. Broderick, Opt. Lett. 20, 1674 (1995).

9. B. J. Eggleton. C. M. de Sterke. and R. E. Slusher, Opt. Lett. 21. 1223 : 1996).

10. N. G. Broderick and C. M. de Sterke, Phys. Rer. E 55, 3634 (1997). 